Sociologie et sociétés

\title{
Système mondial et mouvements nationaux dans les pays industrialisés
}

\author{
L'exemple Québec/Canada
}

\section{World System and National movement in Industrialized Countries}

\section{The Case of Quebec/Canada}

\section{Arnaud SALES}

Volume 11, numéro 2, octobre 1979

Développement national et économie mondialisée

URI : https://id.erudit.org/iderudit/001677ar

DOI : https://doi.org/10.7202/001677ar

Aller au sommaire du numéro

\section{Éditeur(s)}

Les Presses de l'Université de Montréal

\section{ISSN}

0038-030X (imprimé)

1492-1375 (numérique)

Découvrir la revue

Citer cet article

SALES, A. (1979). Système mondial et mouvements nationaux dans les pays industrialisés : l'exemple Québec/Canada. Sociologie et sociétés, 11(2), 69-96. https://doi.org/10.7202/001677ar

\section{Résumé de l'article}

Cet article met en rapport deux mouvements apparemment contradictoires qui contribuent à donner sa configuration au monde contemporain : d'une part un mouvement de globalisation, qui conduit à parler de système capitaliste mondial, d'autre part un mouvement de différenciation nationale qui, sortant du Tiers-monde, touche maintenant les grands pays industrialisés et les menace de morcellement. S'appuyant sur l'exemple Québec/Canada, l'auteur montre d'abord comment l'intégration continentale dépendante,

l'internationalisation et la concentration qui constituent les dimensions principales du processus de globalisation, empêchent le Canada de se maintenir comme champ économique autonome et surtout comme unité pertinente pour résoudre la question du redéploiement industriel et du développement régional inégal. C'est dans ce contexte que le mouvement national québécois, fondé d'abord sur la lutte contre des structures de domination/subordination entre deux nations, pose à travers la revendication d'une "souveraineté-association", la double question de la différenciation nationale et de l'intégration au système global pour contrer la marginalisation, l'affrontement pour la réorganisation du pouvoir social ayant surtout lieu entre les couches dirigeantes de l'État québécois et la bourgeoisie canadienne associée aux sommets de l'État fédéral. 


\section{Système mondial et mouvements nationaux dans les pays industrialisés : l'exemple Québec-Canada*}

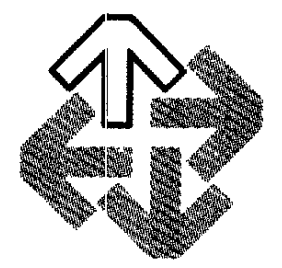

ARNAUD SALES

«Vous apparaitrez dans les États-Unis d'Amérique, une des nations des temps jadis, correspondant à une division politique dont le but n'a pas été établi de façon certaine. Un des buts de votre expédition sera justement de déterminer pourquoi la race humaine de l'époque se dispersait en une multitude de nations au lieu d'avoir un gouvernement unique», dans Fredric Brown, Fantômes et farfafouilles, Paris, Denoël, 1963, p. 126.

Le $\mathrm{xx}$ e siècle, à travers ses multiples péripéties, aura, sur le plan international, été marqué par deux grands mouvements : un mouvement de globalisation, d'accentuation des interdépendances qui conduit à parler de système mondial et un mouvement de recomposition de la géographie politique du globe caractérisé par l'émergence d'une multitude de nouveaux États, et donc par le morcellement, la dislocation, la balkanisation.

D'un côté, le monde contemporain a vu plus que tout autre, se développer de vastes ensembles dotés d'une puissance économique, politique et adminis-

* Version remaniée de la communication au Colloque sur «les Implications sociales et politicues du nouvel ordre économique international» organisé par le Comité de recherche «Économie et société » de l'Association internationale de sociologie, Fondation Rockefeller à Bellagio, Italie, 24-28 avril 1979. La version anglaise originale paraîtra dans Makler, Martinelli et Smelser, édit., The New Transnational Economic Order, Sage Publications. 
trative, militaire, scientifique et culturelle encore jamais atteinte, ce qui conduit à penser que la modernité est étroitement associée à la concentration, à l'intégration, à l'internationalisation. D'un autre côté, l'apparition de la «patrie planétaire", du Global village semble, à travers un mouvement de différenciation et de recherche d'identité, appeler dans le même temps l'apparition de «petites patries». Les luttes de libération nationale en démembrant les anciens empires coloniaux ont depuis 1945, donné naissance à une centaine d'États multipliant ainsi leur nombre par 3. La transformation de la planisphère politique ne semble pourtant pas encore achevée puisque, sortant de la zone du Tiers monde, des mouvements nationaux ou régionaux se sont levés, cette fois, dans bon nombre de pays industrialisés. Certains sont encore embryonnaires, mais d'autres sont suffisamment puissants pour faire trembler des systèmes de frontières. Ainsi, quelques grands États comme la Grande-Bretagne, le Canada, la France pourraient un jour se trouver morcelés ou à tout le moins réorganisés avec, notamment, une capacité de décision économique centralisée beaucoup plus faible.

La légitimité acquise par les luttes de libération nationale dans les pays de la périphérie, ne s'est pas automatiquement décalquée sur les mouvements nationaux ou régionaux (basque, écossais, occitan, jurassien, flamand ou québécois) qui revivent dans les pays industrialisés. Sans doute reconnaît-on le particularisme culturel de ces régions (dimension folklorique?), sans doute reconnaît-on aussi leur sous-développement relatif et même l'existence d'un certain colonialisme intérieur aussi bien économique que culturel. Mais les manifestations de ces mouvements et de ces luttes engendrent, à priori, scepticisme et même agacement. Pourtant, depuis quelques années, leur signification globale est sortie de l'ombre et de la marginalité. La France, le Canada, la Grande-Bretagne, l'Espagne, la Belgique, la Suisse cherchent d'abord à réprimer, puis à contenir et à la dernière limite, à négocier avec ces mouvements nationaux qui s'incarnent de plus en plus dans des partis organisés.

On ne peut aujourd'hui prédire l'impact global à long terme de la «reviviscence des mouvements nationaux dans les pays industrialisés ${ }^{1}$. Mais la question de la dislocation possible de quelques grands États nations reste posée. Notre intention ici n'est évidemment pas d'en traiter les multiples facettes. Nous tenterons seulement de voir si cette tendance à la fragmentation politique n'est en définitive qu'un corollaire de la globalisation qui profiterait, en fait, de l'éclatement d'États nations de puissance moyenne en mini-pseudo-États sans réel pouvoir, aussitôt à la merci des multinationales, puissances du capital privé transnational dont les dimensions, on le sait, se comparent avantageusement à des pays comme la Suisse ou le Vénézuela, si l'on se risque à comparer les ventes annuelles et le PNB? Ainsi la recherche d'une souveraineté nationale, dont les ressorts fondamentaux se trouvent pourtant au moins autant dans le refus de la subordination économique que dans la spécificité culturelle, n'aboutirait, en réalité, qu'à l'obtention d'une série de symboles politiques d'opérette tandis que la dépendance économique se renforcerait à cause des faibles

1. Du titre d'un colloque international organisé par l'Université du Québec à Montréal les 4,5 et 6 avril 1979. 
dimensions de l'espace ainsi morcelé et du manque relatif de pouvoir de ce nouvel État national dans une économie mondialisée. La montée de mouvements nationaux serait-elle donc aussi illusoire? C'est ce qu'il convient de commencer à examiner ici en s'appuyant sur l'exemple Québec-Canada.

\section{LA GLOBALISATION}

La réalité d'un système mondial n'est pas nouvelle et l'on pourrait à la suite de Braudel ou de Wallerstein dater son émergence à la fin du XV $\mathrm{XV}^{\mathrm{e}}$ siècle. $\mathrm{Ce}$ qui est nouveau cependant, c'est le processus actuel d'intégration qui le caractérise. Depuis la fin des années 60 , de très nombreux chercheurs ont focalisé leurs analyses sur la mise en place de ce nouveau système global encore en voie de constitution, que ce soit à travers l'étude du procès d'internationalisation du capital, du développement des firmes multinationales, des tendances à la transnationalisation, et plus généralement encore d'une réflexion sur le nouveau capitalisme mondial (Vernon, 1971; Amin, 1971 et 1973 ; Palloix, 1972; Stopford et Wells, 1972; Wilkins, 1970 et 1974; Barnet et Muller, 1974; Furtado, 1975; Michalet, 1976). Le mouvement de globalisation à l'échelle mondiale se traduit notamment par un processus d'intégration généralisée, une structure du pouvoir économique et politique de plus en plus concentrée et une tendance à la transnationalisation qui se traduit sous des formes diverses. Ces dimensions ont toutes un effet sur notre objet et il faut donc en rappeler brièvement le contenu.

Le processus d'intégration se réalise à trois niveaux spécifiques. D'abord au centre du système capitaliste où des liaisons de plus en plus étroites se sont établies entre les grandes économies nationales à travers des échanges considérables de capitaux, de produits, d'hommes hautement qualifiés et de connaissances scientifiques et techniques. Il en résulte une unification, une homogénéisation de l'espace économique (même si de vastes régions sont laissées pour compte), et l'apparition de zones décisionnelles ${ }^{2}$ échappant de plus en plus aux États nationaux (Furtado, 1975).

Le processus d'intégration se réalise aussi de plus en plus entre le Centre et la Périphérie, le Nord et le Sud, s'appuyant sur le développement inégal des différents pays. Pour des raisons structurelles ${ }^{3}$, le capitalisme ne peut survivre

2. Celso Furtado écrit «Tout se passe comme si une nouvelle dimension était apparue, qui échappe aux formes d'action codifiées par les gouvernements sur le plan national ou sur le plan international. Bref à l'intérieur du cadre institutionnel actuel, les gouvernements n'ont pas la possibilité de coordonner l'action que tout un ensemble d'agents puissants exercent dans le système capitaliste. S'il existe une coordination, elle se fait dans le cadre des oligopoles et des consortiums financiers où les gouvernements se manifestent par la pression qu'ils exercent sur tel agent». Dans «Le capitalisme post-national», Esprit, avril 1975, p. 490.

3. Charles-Albert Michalet indique à ce sujet que «la problématique majeure, avec l'apparition de l'économie mondiale, n'est plus celle de la réalisation de la valeur, elle est devenue celle de la création elle-même. Ceci ne signifie pas que la nouvelle contradiction chasse l'ancienne... Mais l'apparition d'un problème au niveau de la création, manifestation de la sur-accumulation du capital, a exigé du capitalisme qu'il revête de nouvelles formes pour sa reproduction. Dans la mesure où le haut niveau de développement des forces productives rend problématique d'accroître les taux de plus-value relative, la solution consiste à rechercher des zones où le taux de plus-value est plus élevé. Il s'agit donc de déplacer le processus productif vers des régions moins développées tout en gardant évidemment sa maitrise». Dans le Capitalisme mondial, p. 225. 
sans délocaliser la production et donc sans lancer la force de travail du Tiers monde dans la production industrielle, l'intégrant de façon nouvelle au système mondial, mais toujours dans une situation de dépendance (Michalet, 1976). Comme on le verra plus loin, ce processus a, évidemment, des conséquences considérables sur l'évolution de la structure industrielle des pays du centre qui doivent tous chercher à reconvertir leurs branches industrielles à faible productivité.

Cette intégration des espaces économiques s'accompagne d'une intégration et d'une centralisation au sein même des appareils qui matérialisent les transformations dont nous venons de parler. Les entreprises multinationales, en effet, dépassent les cadres traditionnels de l'organisation par pays, intègrent leurs activités clés entre les filiales et donc centralisent, au niveau de la direction internationale de la firme, la définition des objectifs, des stratégies et des politiques qui appartenaient autrefois pour l'essentiel à des entreprises locales ou nationales indépendantes. La stratégie de mise en valeur du capital n'est pas réalisée de façon fragmentée et autonome par chaque filiale, mais au niveau de la firme dans son ensemble.

Le processus d'intégration d'activités géographiquement très dispersées et géographiquement différenciées s'accompagne, par conséquent, d'un mouvement de centralisation et de concentration de la propriété et du pouvoir à l'échelle internationale, mais aussi au niveau de l'ensemble de l'organisation sociale puisque l'entreprise est un de ses éléments les plus fondamentaux. Cette centralisation accentuée par le progrès des systèmes d'information et de communication implique nécessairement que soient subordonnés au système global de la FMN, les entreprises tronquées que sont les filiales, les entreprises locales sous-traitantes, la main-d'œuvre qui ne peut lutter qu'à un niveau très local, et plus généralement les intérêts nationaux, les cultures et les modes de vie.

Sur le plan intérieur, la concurrence internationale force la concentration par centralisation du capital, et les États nationaux du centre ont, dans la plupart des cas, dû intervenir pour faciliter la réorganisation, la «rationalisation» de branches industrielles stratégiques complètes, agissant comme des catalyseurs de la formation d'une structure de plus en plus monopolisée. Ce mouvement renforce évidemment la fraction monopoliste de la bourgeoisie intérieure qui cherche alors à s'internationaliser. Cependant, comme je l'ai montré pour le Québec ${ }^{4}$, les fractions du capital s'incarnent socialement, non seulement au sein d'une classe, mais aussi à travers des groupes nationaux et ethniques spécifiques. Dans la mesure où certains de ces groupes sont mieux représentés dans la structure du pouvoir économique et politique, il est facile de comprendre que la concentration s'effectue à leur profit et au profit des régions où se trouvent les bases principales de leur pouvoir.

Cette centralisation et cette intégration s'accompagnent enfin d'une tendance à la transnationalisation propre à l'ensemble du nouveau système mondial. Celle-ci se repère d'abord dans le développement d'une superstructure

4. Arnaud Sales, la Bourgeoisie industrielle au Québec. 
suprarationale qui s'institutionalise formellement ou informellement dans des domaines comme la défense, l'économie, la politique, les finances, mais aussi les professions, les mass media, l'information, la science et la technologie (Sunkel et Fuenzalida, 1979).

Elle se repère évidemment au sein des FMN qui, nous l'avons déjà noté, dépassent les cadres traditionnels des frontières nationales ou régionales pour établir leurs stratégies, leurs modes d'organisation et leurs implantations. Pour l'oligarchie des «firmes globales », l'État nation indépendant est à la fois un obstacle à l'optimisation des ressources à l'échelle mondiale et une catégorie obsolescente inadaptée à la complexité économique et technique de notre univers. Sur le plan idéologique, l'universalisme impérialiste de la grande corporation s'oppose radicalement au nationalisme qui freine, réglemente ou concurrence son extension (Barnet et Muller 1974).

Fourtant, et il est essentiel d'en tenir compte dans toute analyse, les FMN ne peuvent pas être considérées comme de pures institutions transnationales puisqu'elles conservent toujours une base nationale marquée (Michalet, 1976; De Brunhoff, 1976). À cet égard les rapports entre les multinationales et l'État nation sont à la fois conflictuels et complémentaires, donc nécessairement ambigus. Centres de pouvoir principaux des forces sociales dominantes dans nos sociétés, la dialectique de leurs rapports est extrêmement importante dans la formation du nouveau système global puisqu'elle est soustendue par ur jeu complexe de luttes sociales à la fois internes et externes. En fait, si l'on considère plus généralement le réseau des rapports tissé entre le capital autochtone, le capital étranger et le pouvoir d'État, il convient de reconnaître que ces relations diffèrent profondément d'une société à l'autre selon leur niveau de développement, leur degré de dépendance, mais aussi selon l'état des luttes sociales qui s'y déroulent avec plus ou moins d'intensité selon les époques. De ce point de vue, il est central de réintroduire l'impact des mouvements sociaux qui peuvent peser de façon considérable dans la définition de ces rapports.

\section{LA GLOBALISATION ET L'INTÉGRATION DU CANADA}

\section{L'INTÉGRATION CONTINENTALE DÉPENDANTE}

Il est certain que même si la CEE et le Japon peuvent de plus en plus souvent se poser en concurrents sérieux, les États-Unis occupent encore une position hégémonique dans le système actuel. C'est donc par rapport au géant américain que se réalise d'abord l'intégration des économies du Centre. Cependant cette intégration a une intensité qui varie selon les pays. Or c'est le Canada qui, parmi les pays industrialisés de puissance moyenne, remporte la palme, souvent amère, de cette intégration dépendante. Il suffit, pour en saisir l'étendue, de rappeler que $58 \%$ des actifs et $66 \%$ des profits de l'industrie de fabrication et de transformation sont sous contrôle étranger, que dans l'industrie minière, la proportion est de $68 \%$ pour les actifs et de $72 \%$ pour les profits, 
et que ce contrôle s'effectue à $80 \%$ sous l'égide de firmes multinationales américaines.

Les richesses naturelles de cet immense territoire ont fait du Canada le gros fournisseur de produits de base de l'Amérique du Nord qu'il s'agisse du bois, du minerai pour de nombreux métaux ${ }^{5}$ et, dans une certaine mesure, du pétrole. Compte tenu de l'histoire économique canadienne, il n'est pas très surprenant de constater que le Canada représente environ $50 \%$ des investissements directs américains à l'étranger dans ce secteur.

La dépendance canadienne à l'égard du capital américain est donc considérable. L'intégration continentale nord-américaine se lit à bien d'autres niveaux, et en particulier au niveau des échanges commerciaux, puisque les trois quarts des produits canadiens vendus à l'étranger vont aux États-Unis. Cependant, il s'agit généralement de produits primaires ou semi-finis, le Canada important de nombreux produits manufacturés. Avec les nouveaux accords du GATT, cette intégration devrait se poursuivre puisque $80 \%$ des exportations canadiennes entreront aux États-Unis en complète franchise. Par ces mesures, on s'achemine donc progressivement vers une zone de libre échange nord-américaine, sinon un marché commun Canada-États-Unis-Mexique.

Cette dépendance à l'égard des États-Unis n'est pas seulement l'effet d'une stratégie dynamique d'investissement direct des multinationales américaines. Il s'agit aussi d'une dépendance induite. À cause de ses antécédents coloniaux, la bourgeoisie intérieure canadienne a toujours été caractérisée par la dominance des fractions commerciale et bancaire (de type britannique), la fraction industrielle ayant un poids relatif beaucoup plus faible, ce qui explique, en partie, les difficultés du développement industriel autochtone et la pénétration de l'investissement direct américain dans l'industrie ${ }^{6}$ (Naylor, 1972, 1975; Clement, 1975; Sales, 1979). Aussi aujourd'hui trouve-t-on 57 firmes étrangères parmi les 100 premières entreprises du Canada. Parmi les 200 plus importantes firmes industrielles, figurent 91 filiales d'entreprises américaines classées dans la liste des 500 de la revue Fortune.

\section{L'INTERNATIONALISATION DU CAPITAL CANADIEN}

Cette dépendance excessive du Canada qui freine l'accumulation «nationale» de façon considérable puisque le rapatriement des dividendes et intérêts s'élève à 2,5 milliards de dollars par an, n'empêche pas le capital monopoliste

5. Le Canada se place au premier rang de la production mondiale pour le nickel, le zinc, l'amiante, au deuxième rang pour la potasse, au troisième rang pour le plomb et au quatrième rang pour le cuivre.

6. L'induction de la dépendance par la bourgeoisie bancaire canadienne apparaît dans l'accueil à bras ouverts qu'elle a toujours réservé aux filiales d'entreprises étrangères au détriment des industriels locaux. "Des sociétés multinationales dont le siège social est situé au Royaume-Uni ou aux États-Unis ont eu, en outre, un accès facile aux grandes sources de capitaux, à bien meilleur compte qu'au Canada. Quand la filiale canadienne d'une société multinationale décide de réunir ses capitaux au Canada, les institutions financières canadiennes sont plus disposées à lui consentir des prêts qu'aux sociétés nationales de taille semblable et souvent à des taux plus avantageux », dans Rapport de la Commission royale d'enquête sur les groupements de sociétés, 1978, p. 219. 
canadien de s'internationaliser. Moore Corporation, Inco, Massey-Ferguson, Northern Telecom comptent des investissements très importants à l'étranger. Plusieurs indicateurs montrent que le mouvement s'est intensifié au cours des dernières années et la Commission royale d'enquête sur les groupements de sociétés indiquait, par exemple, que jusqu'en 1976, les investissements directs du Canada à l'étranger ont dépassé les investissements directs venus de l'extérieur (les entreprises étrangères se finançant dans la plupart des cas directement au Canada). Les banques ont été particulièrement actives dans ce processus à partir des années 60. Ainsi, "les passifs et les avoirs en devises étrangères des banques à charte ont augmenté respectivement de 667 et de $688 \%$ de décembre 1966 à décembre $1976^{7} \ldots$ Environ le quart du produit des cinq plus grandes banques à charte canadiennes (soit $90 \%$ des actifs bancaires au Canada) provient de leurs activités à l'étranger... (tandis que) les avoirs étrangers constituent $30 \%$ des avoirs globaux ${ }^{8}$. . Rappelons aussi que le total des succursales des banques à charte canadiennes à l'étranger s'élevait au 31 mai 1977 à 497.

Tableau I

Succursales des banques à charte canadiennes à l'extérieur du pays au 31 mai 1977

\begin{tabular}{lc}
\hline & $\begin{array}{c}\text { Nombre de } \\
\text { succursales }\end{array}$ \\
\hline Royaume-Uni & 28 \\
États-Unis & 70 \\
France & 8 \\
Allemagne de l'Ouest & 7 \\
Bahamas & 52 \\
Barbade & 26 \\
Guyane & 11 \\
Mexique & 4 \\
Amérique centrale et Amérique du Sud & 26 \\
République dominicaine & 22 \\
Îles Vierges (américaines et britanniques) & 7 \\
Porto Rico & 16 \\
Trinidad et Tobago & 33 \\
Jamaique (et les îles Cayman) & 93 \\
Autres pays des Indes occidentales & 31 \\
Autres pays européens & 16 \\
Asie (y compris le Moyen-Orient) & 42 \\
Australie & 5 \\
Total & 497 \\
\hline
\end{tabular}

Source: Association des banquiers canadiens. Dans Commission royale d'enquête sur les groupements de sociétés. Les banques à charte sont représentées par des succursales, des agences, des bureaux de mandataires ou par des succursales de filiales.

Wallace Clement, dont on connaît les travaux remarquables sur l'intégration du pouvoir économique à l'échelle continentale, distingue à ce sujet deux formes

7. «Cette expansion est attribuable à l'évolution du système de l'euromonnaie au sein duquel les banques canadiennes se disputent avec les banques étrangères les gros dépôts de dollars américains. Elles reprêtent ces dépôts à des résidents comme à des non résidents (y compris les banques étrangères), ou encore elles les investissent dans des valeurs en dollars américains » dans Commission royale sur les groupements de sociétés, p. 263.

8. Commission royale d'enquête sur les groupements de sociétés, p. 263. 
d'investissement canadien à l'étranger. La première provenant essentiellement des secteurs du transport, des services et surtout des banques et des institutions financières, où la bourgeoisie intérieure prédomine, peut être considérée comme le «véritable» investissement canadien à l'étranger. La seconde, qualifiée de «go-between investment », permet à des filiales américaines de contrôler, à partir du Canada, d'autres filiales dans le reste du monde. Ainsi $36 \%$ (soit $\frac{\$ 1820 \mathrm{M}}{\$ 9307 \mathrm{M}}$ ) de l'investissement direct canadien à l'étranger en 1974, relevait de la deuxième forme (Clement, 1978).

\section{LES PARADOXES DE LA STRUCTURE ÉCONOMIQUE}

Plusieurs paradoxes peuvent par ailleurs être notés quand on examine les rapports entre la présence de capital étranger, la concentration et l'internationalisation du capital autochtone. En premier lieu, il faut indiquer que la superconcentration est la règle dans l'économie canadienne. Dans le secteur bancaire, cinq grandes banques se partagent $90 \%$ des actifs du secteur. Dans l'industrie, la concentration est nettement plus forte qu'aux États-Unis, à tel point que «la concentration globale au Canada est d'environ le double de celle des États-Unis, tandis que les tendances générales dans les deux pays sont virtuellement les mêmes ${ }^{9}$.

\section{GRAPHIQUE 1}

Pourcentage du total national de l'actif et des ventes attribuable aux 100 plus importantes* entreprises non financières, Canada et États-Unis, 1966, 1971 et 1975
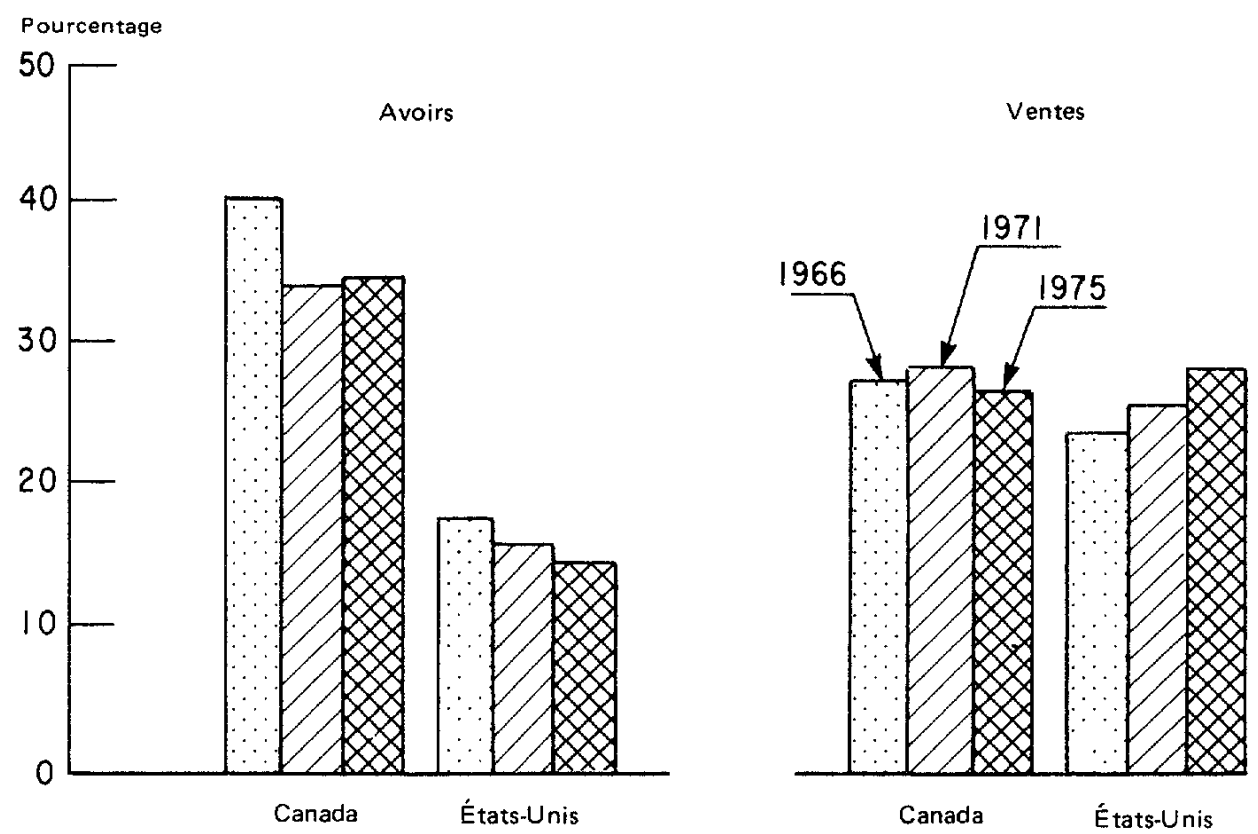

Source: Recherche de la Commission royale d'enquête sur les groupements de sociétés.

* Classées suivant les ventes.

9. Commission royale d'enquête sur les groupements de sociétés, p. 33. 
En second lieu, les investissements étrangers directs ne semblent pas avoir eu d'effet sur le niveau de concentration. Par contre, la structure oligopolistique du marché américain ou international a souvent été transplantée par les investissements des multinationales constituant cet oligopole, d'où la « reproduction en miniature " caractéristique de nombreuses branches industrielles au Canada ${ }^{10}$.

En troisième lieu, la superconcentration n'implique pas que la taille des entreprises canadiennes soit suffisante pour affronter la concurrence internationale ". Par ailleurs, le capital industriel autochtone se trouve souvent, comme on le verra un peu plus loin, dans des secteurs peu productifs et de plus en plus concurrencés. Les nouvelles structures tarifaires, l'intensification généralisée de la concurrence étrangère et les effets de la délocalisation des industries de main-d'œuvre dans les pays sous-développés forcent en fait à la rationalisation, c'est-à-dire plus exactement à une accentuation de la concentration. La conclusion de la Commission royale est claire à ce sujet:

Dans de nombreux pays, les plus grandes entreprises de certains secteurs ont été poussées à fusionner pour devenir plus concurrentielles sur le plan international. S'il est vrai que pour produire efficacement et faire face à la concurrence sur les marchés, il est nécessaire, comme cela semble le cas dans bien des industries, d'avoir des entreprises de grande taille, les tendances à vouloir réduire la concentration, au Canada, en limitant la taille des entreprises ne feront que diminuer le pouvoir des sociétés canadiennes sur les marchés internationaux. De mếme, si les grandes entreprises canadiennes peuvent acquérir et acquièrent effectivement une taille « internationale», il pourra fort bien s'ensuivre une augmentation considérable de la concentration des entreprises au Canada ${ }^{12}$.

\section{LES RÉPERCUSSIONS SUR LA SOCIÉTÉ CANADIENNE}

L'impact de ces mouvements sur la société canadienne est évidemment considérable. En fait, l'intégration de plus en plus forte du Canada à l'économie américaine par le biais du capital et des marchés, le manque de contrôle du Canada sur son industrie de fabrication et sur ses ressources, et donc sa sous-a.ccumulation relative, la prééminence des relations maisons mères/filiales sur les objectifs économiques du pays (illustrée encore récemment par le détournement de quantités importantes de pétrole destinées au Canada par Exxon N.Y. au détriment de sa filiale Imperial Oil et surtout de l'économie de l'Est du Canada), le sous-développement scientifique et technologique par monopolisation de la recherche à l'extérieur du pays, conduisent à un affaiblissement du développement national puisque l'entité canadienne, dans sa globalité, tend à perdre de plus en plus de sa capacité à se transformer par ses propres moyens. Ainsi dans de nombreux domaines aussi bien économiques que politi-

10. Commission royale d'enquête sur les groupements de sociétés, p. 219.

11. "Les sociétés canadiennes les plus importantes sont plus grandes par rapport à l'économie carladienne que ne le sont les sociétés américaines les plus importantes par rapport à l'économie des États-Unis; mais en termes absolus, nos sociétés sont beaucoup plus petites (les données indiquent qu'elles correspondent environ au cinquième de la taille moyenne) que les plus grandes entreprises américaines", dans Commission royale d'enquête sur les groupements de sociétés, p. 33 .

12. Ibid., pp. $455-456$. 
ques ou culturels, le Canada ne peut guère jouer qu'un rôle de «second violon des États-Unis». Mais les choses vont encore plus loin car ces facteurs associés au manque de maîtrise sur les implantations étrangères ${ }^{13}$ et à la superconcentration industrielle, commerciale et bancaire favorisent l'apparition d'une économie éclatée sur le plan régional.

Le manque de maîtrise sur les implantations étrangères se reflète dans la distribution de l'investissement étranger qui prédomine sur le capital canadien en Ontario et en Alberta, accentuant ainsi les disparités régionales. Les effets de la superconcentration se reflètent, quant à eux, dans une stratégie de développement fédérale fondée sur de très grands projets liés à l'énergie et à son transport menés par les grandes entreprises concentrées, indépendamment de leur nationalité, et bien entendu avec les garanties de l'État (sables bitumineux de l'Athabasca, oléoducs et gazoducs du Grand-Nord), mais sans que cette stratégie prenne réellement en compte la dimension régionale. Quant à la concentration bancaire, et plus largement financière, elle a certainement nui au développement régional à cause des contraintes très lourdes imposées par cette structure très centralisée qui a toujours tendance à asphyxier financièrement les petites et moyennes entreprises dont la vocation est le plus souvent locale. Plus largement, l'unification monétaire des régions canadiennes, l'existence d'une banque centrale unitaire au lieu d'un système fédéral de banque centrale, impose un «style unitaire de gestion» de la politique monétaire qui se soucie bien plus des réactions du capital monopoliste que des particularités régionales ${ }^{14}$. Par ailleurs, la centralisation du capital a son pendant géographique puisque Toronto au détriment de Montréal tend à regrouper les grands centres de décision financiers et industriels.

Il faut mentionner enfin l'impact régional des bouleversements des rapports Centre-Périphérie qui menace des pans entiers de l'économie de nombreux pays industrialisés et qui implique donc une gigantesque mutation industrielle qui souvent touche des régions très spécifiques: le Québec en est un bon exemple puisque $25 \%$ de sa main-d'œuvre est concentrée dans les secteurs «mous» du textile, de la bonneterie, du vêtement et du meuble. Or c'est là que se pose, de façon complexe, la question régionale élargie en question nationale à cause des relations de domination/subordination nouées entre les deux nations anglaise et française, fondatrices du Canada. Ce n'est pas tout à fait un hasard, en effet, si les industries de main d'œuvre liées à l'existence d'une réserve de cheap labor se sont concentrées au Québec.

13. Voir à ce sujet, Ray, D. M., «Regional Economic Development and the Location of U.S. Subsidiaries», communication à l'Incentive Location and Regional Development Conference, 1975 et Ray, D. M., "The Location of American Subsidiaries in Canada», Economic Geography, vol. XLVII, n 3 , juillet 1971, pp. 389-400.

14. Vély Leroy écrit à ce sujet «Tout compte fait, l'élaboration courante de la politique monétaire au Canada conserve encore et l'esprit et la démarche des années '30, que sont du reste venus renforcer le second conflit mondial et les excès de la centralisation. Par là, nous entendons qu'elle participe du style unitaire de gestion. Et comme il lui faut des appuis dans les sphères économiques et financières afin que les mesures décidées soient tournées en faits, il va de soi que le comportement des milieux d'activité les plus réceptifs aux signaux de la Banque centrale sera déterminant dans le choix des dispositions à prendre, souvent au mépris des particularités régionales. Or, il n'est pas toujours vrai que celles-ci doivent être sacrifiées nécessairement", dans la Question monétaire en rapport avec le Québec, p. 274. 


\section{LES CONTRADICTIONS DE LA TRANSNATIONALISATION}

Dans la mesure où la bourgeoisie monopoliste cherche à s'étendre à l'échelle internationale, l'idéologie nationaliste au niveau de l'État central tend à refluer, à se désintégrer puisqu'elle est alors un obstacle à la stratégie internationale des fractions intérieure et compradore de la bourgeoisie monopoliste.

Malgré les volumineux rapports Watkins et Gray sur les effets néfastes de l'investissement étranger, l'agence de «tamisage», en trois ans, a laissé passer 2,5 milliards d'actifs sous contrôle étranger acceptant $88 \%$ des demandes. Jean Chrétien, ex-ministre de l'industrie se flattait de n'avoir rejeté que 10 des 160 projets qui défilèrent sur son bureau. Mel Watkins, à l'occasion du $10^{\mathrm{e}}$ anniversaire de son rapport, rappelait qu'Ottawa, traditionnellement, a toujours été du côté des multinationales contre les provinces, par exemple, au moment de la nationalisation de la potasse par le gouvernement de la Saskatchewan où l'État fédéral s'est associé au cartel des multinationales dans la guérilla juridique devant la Cour Suprême. L'achat de l'Asbestos Corp. par Québec entraînera sans doute le même type d'intervention. On pourrait multiplier les exemples indiquant l'absence de volonté politique de l'État central sur cette question. Ainsi en mars 1979, les députés fédéraux, en Commission parlementaire, invitaient à une extension des banques étrangères au Canada pour résoudre le problème de la concurrence dans le système bancaire.

En fait, la boucle de l'intégration continentale dépendante du Canada est en train de se boucler lorsque la Commission royale d'enquête sur les groupements de sociétés, liant la solution du problème du pouvoir économique sur les affaires canadiennes exercé par des multinationales et celui de la «rationalisation» nécessaire pour affronter le marché mondial, conclut qu' «au lieu de racheter les entreprises détenues par des étrangers, il serait préférable de prévoir les moyens d'encourager la fusion des entreprises tant étrangères que nationales faisant partie d'une même industrie, en vue de créer des entités plus grandes et plus efficaces, et de recourir à des lois qui l'emporteraient sur toute opposition anti-trust de la part des Etats-Unis et d'autres pays ${ }^{15}$ ".

Si cette intégration globale et mal contrôlée réussit parfaitement au profit d'un petit nombre de centres de pouvoir dont beaucoup se trouvent de l'autre côté de la frontière, les disparités entre les provinces canadiennes ne cessent de s'accroître et des tendances centrifuges se manifestent non seulement au Québec, mais aussi en Alberta et en Colombie britannique. Vély Leroy souligne que

contre toute attente, la «balkanisation» de l'espace économique canadien se passe en sourdine. Elle est depuis longtemps une réalité dans maints domaines. À l'origine de ces développements, il existe bien sûr, des impedimenta. Ainsi, la moins que parfaite mobilité des hommes est une cause agissante... mais il y a, en plus, l'éveil des populations locales et leurs aspirations au bien-être; aussi des gouvernements locaux de plus en plus soucieux de leurs responsabilités dans la sphère économique. Ainsi assiste-t-on à la montée d'un courant protectionniste à l'intérieur même des frontières communes et qu' exacerbent des politiques gouvernementales, des codes de profession, des législations, des décrets, des droits déguisés

15. Commission royale, p. 236. 
à l'importation de marchandises ou de services en provenance d'autres régions du pays. La fiscalité elle-même n'est pas hors de cause ${ }^{16}$.

La grande bourgeoisie canadienne, qui a toujours su profiter des déséquilibres régionaux, n'a pourtant aucun intérêt à la fragmentation politique du Canada; c'est pourquoi elle joue à fond la carte de l'unité canadienne qui lui assure un hinterland de grande dimension, où elle peut facilement s"approvisionner et qui, jusqu'à présent, ne lui a guère imposé de contraintes. Une intégration continentale sans frontière affaiblirait son pouvoir politique en la provincialisant. La bourgeoisie compradore elle-même se fondrait dans les cadres supérieurs ordinaires des grandes multinationales américaines. De la même manière, toute réorganisation plus décentralisée du système fédéral actuel pose la question de la réorganisation du pouvoir économique au Canada, et donc de la grande bourgeoisie qui pourrait se voir concurrencée ou mise en question par des États «provinciaux» dotés de leviers économiques beaucoup plus importants qu'aujourd'hui. Il suffit pour cela de penser aux implications de la réorganisation du système bancaire et monétaire.

Ceci indique d'ores et déjà que si la tendance à la dislocation politique des États industrialisés peut être considérée comme une dimension du processus de globalisation, elle est loin de pouvoir être interprétée, ainsi que nous nous le demandions au début de ce texte, comme son simple corollaire. Comme on va le voir dans le cas du Québec, la grande bourgeoisie continentale a peu d'intérêts à ce que les frontières canadiennes d'aujourd'hui soient transformées car la dislocation signifie immédiatement la réorganisation sur le plan régional et donc la menace d'organisation du capital sur une base plus étatique, plus nationaliste et plus contraignante. Ceci est vrai pour la bourgeoisie intérieure, mais aussi pour la bourgeoisie américaine qui, satisfaite par la sécurité du Canada actuel, n'a jamais été véritablement gênée par ses frontières qu'elle a souvent considérées comme purement formelles, ainsi qu'en témoigne l'organisation administrative des entreprises où les activités canadiennes ont souvent été intégrées à leur division, non pas internationale, mais domestique.

C'est pourquoi, de façon générale, la désagrégation des grands États nations au profit d'unités régionales souveraines apparaît aux stratèges occidentaux, comme nuisible aux intérêts des grandes bourgeoisies en place, dangereuse dans le système de sécurité politico-militaire occidental, qu'il faut interpréter et présenter comme un combat d'arrière-garde dramatiquement illusoire pour les forces sociales et les nations subordonnées à la recherche d'une souveraineté.

\section{MOUVEMENTS NATIONAUX ET MORCELLEMENT DES GRANDS ÉTATS}

La tendance à la fragmentation n'est pas le produit du seul processus de globalisation. En effet, même si les différents facteurs que nous venons de mentionner conduisent à un affaiblissement et à une désarticulation du développement national qui va au-delà de la division spatiale qu'a toujours impliquée

16. Vély Leroy, la Question monétaire en rapport avec le Québec, p. 309. 
l'accumulation capitaliste ${ }^{17}$, qui va donc au-delà du développement inégal intra-national, la tendance à la fissuration, ou carrément à la fragmentation d'États nations est aussi le «produit » de luttes sociales propres à chaque société considérée. À cet égard, l'observation de la formation du nouveau système capitaliste mondial ne peut se faire du seul point de vue des effets de la globalisation. et de l'intégration, indépendamment d'une analyse de la tendance à la différenciation nationale qui se manifeste, d'abord, par le regain ou l'éclosion de mouvements nationalitaires. Il s'agit là d'une question extrêmement complexe que l'on ne peut avoir la prétention de traiter en quelques paragraphes.

Trois remarques peuvent cependant être faites à ce sujet. La première pour indiquer qu'un mouvement national ne se réduit évidemment pas aux luttes contre le développement inégal intra-national, à la seule quête d'un pouvoir accru au niveau régional, au régionalisme. La deuxième touche aux concepts mêmes de nation et de construction nationale. La nation est d'abord une idée, une représentation que les agents sociaux se font de l'ensemble qu'ils constituent à partir d'une communauté d'histoire et de culture. Elle relève donc d'abord de l'idéologie. Cependant, l'analyse de la nation est pratiquement indissociable de l'analyse du pouvoir qui est une des conditions de son existence. C'est pourquoi, on ne peut s'arrêter, comme le font plusieurs auteurs, à la seule dimenision idéologique du fait national, qui, en fait, s'incarne, se matérialise à travers le processus de construction nationale et finalement la constitution d'un État.

ILes liens que l'on peut établir entre la nation et le pouvoir sont multiples. Cependant, leur spécificité structurelle se manifeste de façon décisive lorsque l'on analyse les rapports de domination/subordination qui peuvent s'établir entre des groupes nationaux différents : occupation armée, colonisation, assimilation. Ce qui est nouveau avec les protestations et les mouvements nationalitaires au sein des États industrialisés, c'est qu'ils font apparaître que ce type de rapports existe au sein de sociétés apparemment aussi intégrées et unifiées que, par exemple, la société française. La mise en évidence de ces structures de domination s'est faite de plus en plus systématique et précise au cours des 15 dernières années ${ }^{18}$ : minorisation et marginalisation sur le plan du pouvoir politique (variable selon le caractère centralisé ou décentralisé du système politique), dépossession du capital et des centres (privés ou publics), comme des positions (encadrement et direction) de pouvoir économique ${ }^{19}$, surexploitation de la classe ouvrière, effacement ou mise en infériorité de la langue et de la culture. Ainsi, un peuple se trouve-t-il globalement mis en tutelle et marginalisé.

17. Sur le capitalisme et la division spatiale du travail, voir entre autres Doreen Massey, «Regionalism : Some Current Issues», dans Capital and Class, $\mathbf{n}^{\circ} 6$, automne 1978.

18. A propos du Québec, on peut se reporter à l'article de Paul $\mathbf{R}$. Bélanger et Céline Saint-Pierre, «Dépendance économique, subordination politique et oppression nationale : le Québec 1960-1977», dans Sociologie et sociétés, vol. 10, $\mathrm{n}^{\circ} 2$, octobre 1978. Sur un plan plus descriptif, on ne peut ignorer le travail de défrichage empirique considérable de la Commission royale sur le bilinguisme et le biculturalisme et de la Commission Gendron.

19. Voir à ce sujet A. Sales, la Bourgeoisie industrielle au Québec. 
Dans la plupart des cas, le refus de la domination conduit à une affirmation de l'identité qui se cristallise souvent d'abord sur la langue, mais qui débouche en même temps sur des luttes contre l'oppression nationale, la revendication d'un droit à l'autodétermination et la recherche d'une souveraineté qui peut aller jusqu'à la création et au développement d'un État.

Ce qui fait l'unité d'un mouvement national, c'est la primauté du projet de transformation de la collectivité en sujet. Cependant, comme se pose presque immédiatement la question de «la souveraineté pour quoi?, on voit se greffer au projet principal, des projets subsidiaires de transformation plus ou moins radicale du social, qui vont de l' «alternative» communautaire à une civilisation désintégratrice des communautés naturelles et des personnalités ethniques» (Queré, 1978), au projet socialiste dans ses multiples expressions.

Ceci nous amène à la troisième remarque. À mesure qu'un mouvement national se renforce et s'élargit, et lorsque la question nationale finit par occuper, de façon durable, le devant de la scène politique, l'utopie tend à faire place aux stratégies. Dans cette conjoncture, les rapports entre les forces sociales hétérogènes s'intensifient au sein même du mouvement tandis qu'il affronte de plus en plus directement ses adversaires (État central, grande bourgeoisie qui, entre autres, «réaménage» ses actifs en fonction d'une éventuelle souveraineté, mouvements unitaristes ou fédéralistes, «opinion» internationale). Aussi faut-il compter avec les enjeux sociaux propres à chaque société et avec les luttes des forces intérieures autour du pouvoir politique, mais aussi économique.

L'issue de bon nombre de ces luttes est encore très incertaine. Ainsi, le mouvement occitan, dont les premières manifestations contemporaines datent d'une quinzaine d'années ne progresse que lentement et de façon dispersée; les Gallois disent non à la dévolution et si $51,6 \%$ des Écossais répondent oui, les abstentionistes ont raison de la mise en œuvre du «Scotland Act». Au Québec, même si l'issue du référendum du printemps 1980 apparaît, aujourd'hui encore, aussi incertaine qu'en Écosse, le débat, l'alignement et la polarisation des forces sociales en présence ont considérablement progressés depuis que le Parti québécois, qui encadre étroitement le mouvement nationaliste, dispose du pouvoir d'État provincial. L'élection de novembre 1976 constitue une étape historique puisqu'elle ouvre de façon décisive la crise de l'unité canadienne. Ainsi le mouvement nationaliste se manifeste comme force centrale de la société québécoise et la recherche d'une issue politique ne peut se faire en son absence.

Il faut donc s'attarder un petit peu plus à l'examen de ce mouvement pour comprendre ses orientations fondamentales. Cependant, compte tenu du cadre restreint de cet article, nous n'entrerons pas dans une analyse très détaillée qui impliquerait, notamment, une discussion des très nombreux textes qui ont été produits sur cette question. Nous nous limiterons plutôt au rappel de quelques éléments, nécessaires pour comprendre comment s'est imposée l'option «Souveraineté-association» dont la signification diffère quelque peu d'une option indépendantiste claire et nette puisque l'effort de dislocation est immédiatement assorti d'un projet d'intégration: souveraineté politique liée indissolublement à une forme d'association économique à négocier avec le reste du Canada si l'issue du référendum est positive. 


\section{QUÉBEC/CANADA : LE JEU DE LA DISLOCATION ET DE L'INTÉGRATION}

\section{L'ALLIANCE INDÉPENDANTISTE ET LA SOUVERAINETÉ-ASSOCIATION}

Parce que le projet de transformation de la collectivité en sujet, prime sur tout autre projet de transformation du social dans l'action collective qui caractérise les mouvements nationaux, ceux-ci impliquent généralement des forces sociales hétérogènes. Lorsque se forme un parti regroupant les forces indépendantistes, cette hétérogénéité subsiste donc, même si les éléments les plus radicaux ne s'y joignent pas toujours. L'histoire du Parti québécois illustre, une fois de plus, cette caractéristique qui, certes, fait l'ambiguïté de ces organisations politiques, mais qui, aussi, leur donne, jusqu'à l'obtention du statut d'État souverain, la force nécessaire à une mobilisation populaire sous des formes d'ailleurs très diverses.

La fondation du Parti québécois, qui impose dans le mouvement national l'option Souveraineté-association, date de 1968. Mais dès le début des années 60 , des groupes indépendantistes de type nouveau se donnent comme objectif de liberer la nation québécoise ${ }^{20}$ : En 1960, c'est la fondation du Rassemblement pour l'indépendance nationale (RIN), au départ de centre-gauche qui à mesure de sa radicalisation sociale va se séparer d'abord de ses éléments de droite (1962, Parti républicain du Québec), puis de ses éléments démocrates-chrétiens (1964, Regroupement national, dont le but était de faire du Québec «un État souverain et dérnocratique, de culture française et d'inspiration chrétienne»), et enfin en 1968, de ses éléments «révolutionnaires» (Front de libération populaire «indépendantiste, socialiste et décolonisateur»). On retrouve, par ailleurs, à la gauche du RIN, l'Action socialiste pour l'indépendance du Québec, les revues Parti pris et Socialisme québécois et, bien entendu, le fameux Front de libération du Québec (FLQ). Ces groupes vont jouer un rôle fondamental dans la formation d'une idéologie nationaliste progressiste. Mais leur atomisation limitera leur efficacité politique à court terme.

Cependant, des éléments nationalistes se trouvaient au sein même des partis traditionnels, notamment dans le Parti libéral du Québec. Ces forces n'étaient pas négligeables et elles s'étaient manifestées avec vigueur au cours de la Révolution tranquille, non seulement avec le slogan de «maitres chez nous», mais aussi avec toute une série de mesures visant à faire de l'État provincial, un agent important du développement : nationalisation de l'électricité, formation de la Société générale de financement, création de la Caisse de dépôt et de placement et de la sidérurgie d'État, Sidbec ${ }^{21}$.

20. On peut se reporter sur ce point et sur la formation et l'histoire du Parti québécois à l'excellent article de synthèse de Vera Murray, «Le Parti québécois : les tensions au sein de l'alliance indépendantiste», dans Politique aujourd'hui, $\mathrm{n}^{\circ} 7-8$, 1978. Voir aussi V. Murray, le Parti québécois : de la fondation à la prise du pouvoir, Montréal, Hurtubise HMH, 1976. Voir aussi l'intéressante analyse de Gilles Bourque, dans «La nouvelle trahison des clercs», deuxième partie, dans le Devoir, 9 janvier 1979 , p. 5.

21. Voir à ce sujet Pierre Fournier, «Le Parti québécois et la conjoncture économique au Québec», dans Politique aujourd'hui, $\mathrm{n}^{\mathrm{os}} 7-8,1978$. 
Or, c'est justement au cours du congrès du Parti libéral de 1967 que surgit dans un débat d'envergure, la thèse de la Souveraineté-association, défendue par l'ex-ministre des Richesses naturelles, René Lévesque. Le congrès rejette finalement cette option et René Lévesque, entraînant avec lui un groupe de libéraux, fonde alors le Mouvement souveraineté-association (MSA).

Son apparition sur la scène politique bouleversa complètement le mouvement indépendantiste et éventuellement tout l'échiquier politique du Québec. En effet, pour la première fois dans l'histoire du nationalisme québécois, une personnalité aussi connue du public, prestigieuse, charismatique, se ralliait à l'idée de l'indépendance. En octobre 1968, au moment où fut fondé le Parti québécois qui regroupera bientôt la majorité de ceux qui croyaient à l'indépendance du Québec, René Lévesque représentait la caution morale de cette orientation politique. Il fut le seul à pouvoir unifier les éléments hétérogènes qui gravitaient au Québec autour de cet idéal. Un an et demi plus tard, le Parti québécois, fort d'au moins 55000 membres obtient $23 \%$ des voix aux élections provinciales du 29 avril 1970. Encore 6 ans, et il se retrouve au pouvoir avec $41 \%$ des voix ${ }^{22}$.

Le Parti québécois ne regroupe pourtant pas tous les courants indépendantistes. Sa fondation résulte d'abord de la fusion du Mouvement souverainetéassociation et du Regroupement national auxquels vont se joindre individuellement les militants du RIN qui va se saborder dans ce but. La gauche socialiste, d'ailleurs encore embryonnaire à cette époque et aussi quelque peu divisée sur la question nationale, se démarque par rapport au P.Q. pour se développer sous d'autres formes.

Ainsi, le principal «étai structurant» de la construction nationale, pour reprendre l'expression de E. Sicard, est d'abord moulé sur l'option Souverainetéassociation, modèle prudent de souveraineté limitée que les militants estiment capable de recueillir l'adhésion d'une majorité de la population parce que, à priori, ce changement ne bouleversera pas fondamentalement son mode de vie. Et en fait, les tensions au sein de l'alliance souverainiste du P.Q. ne prennent pas corps sur la base du projet «national», mais plutôt sur celle du projet social et de la démocratie à l'intérieur du parti. Comme l'a très bien démontré Vera Murray

toute l'évolution du P.Q. a été marquée par des tensions constantes entre la tendance des «technocrates» (ralliée autour de René Lévesque) préoccupés avant tout par l'efficacité économique et gouvernementale, et la tendance des «participationnistes» (anciens « rinistes», mais surtout professeurs, étudiants, militants syndicaux, animateurs sociaux qui en étaient à leur premier engagement politique), qui attachaient énormément d'importance aux idées de participation, de mobilisation et aux réformes sociales $^{23}$.

L'impact de ces derniers au niveau du programme et de la forme des structures du parti, inspirées de la constitution de la Ligue communiste de Yougoslavie (Murray, 1978), favorisant par conséquent un parti de militants, a joué un rôle fondamental dans l'obtention d'un appui populaire puisqu'un

22. Vera Murray, «Le Parti québécois : les tensions au sein de l'alliance indépendantiste», dans Politique aujourd'hui, $\mathrm{n}^{\text {os }} 7-8,1978$, p. 58.

23. Ibid., p. 58. 
pourcentage élevé d'ouvriers et d'employés ont accordé leur vote au P.Q. en 1976. Cependant les choix politiques centraux restent entre les mains du courant technocratique qui a, évidemment, un poids considérable dans le gouvernement actuel dont le préjugé favorable aux travailleurs, sans être inexistant, joue, comme le disait Gilles Bourque, à la marge de sa politique.

RÉORGANISATION DU POUVOIR SOCIAL ET ENJEUX DE LA SOUVERAINETÉASSOCIATION

On a vu plus haut que toute réorganisation plus décentralisée du système fédéral actuel pose, du même coup, la question de la réorganisation du pouvoir économique. De ce point de vue, la grande bourgeoisie et l'État canadien ont tout intérêt à contenir la question du Québec dans des limites étroitement linguistiques et culturelles en offrant, par exemple, un bilinguisme et un biculturalisme à l'échelle du Canada qui en plus de se révéler impraticable en dehors du Québec, n'a pas d'effets appréciables, si l'on excepte quelque progrès de la présence francophone dans la fonction publique fédérale. Ceci ne signifie pas que la grande bourgeoisie anglo-canadienne soit prête à accepter le règlement de la question linguistique. La langue est intimement liée à l'organisation du pouvoir social. C'est pourquoi les résistances les plus vives au vote et à l'application de la législation linguistique présentée par le gouvernement péquiste sont venues et viennent encore du milieu des affaires anglophone.

Politiquement d'ailleurs, la Charte de la langue française a une importance considérable. Elle démontre d'abord que la question linguistique peut en grande partie être réglée dans le cadre fédéral actuel. Elle contribue surtout à déplacer les luttes du terrain culturel aux terrains politique et économique, beaucoup plus centraux au niveau de la souveraineté et donc de la réorganisation du pouvoir.

En fait, les enjeux sont de plus en plus économiques et de moins en moins culturels. Dans son message inaugural de mars 1979 à l'Assemblée nationale, René Lévesque l'indique clairement :

Mais à notre humble avis, et je n'apprends rien à personne, toute émancipation véritable devra d'abord passer par l'établissement d'un nouveau rapport de forces dans le domaine économique, et par la souveraineté politique qui seule, pourra rapatrier tous les instruments nécessaires à notre développement. La moitié des impôts des québécois alimentent encore les coffres d'Ottawa. Et cette partie de nos taxes est celle qui affecte le plus directement notre économie, elle donne à un autre gouvernement, au service d'une autre majorité, la marge de manœuvre stratégique et les grands leviers d'intervention qui manquent terriblement au Québec. Il nous faut, le plus tôt possible, nous donner les instruments complets et autonomes d'une politique économique qui soit entièrement à notre service, tout en tenant compte des intérêts que nous partageons avec nos voisins et du maintien de l'espace économique canadien. Cette nouvelle définition de nos rapports exige une transformation majeure des structures politiques ${ }^{24}$.

24. Dans le Devoir, 7 mars 1979, p. 11. 
C'est pourquoi le «Fédéralisme reconstitué» assorti d'une souveraineté culturelle québécoise, proposé par la Commission de l'unité canadienne, tout en représentant, comme la souveraineté-association, une troisième voie entre le statu quo et la souveraineté intégrale du Québec, se trouve encore très loin de la proposition du Parti québécois, notamment parce que les principaux pouvoirs économiques d'État (politique monétaire et de crédit, politique commerciale, stratégie industrielle, défense...) resteraient centralisés par le gouvernement fédéral sans que le Québec puisse intervenir directement, d' «égal à égal » pour reprendre le slogan du Parti québécois. Le règlement de la question nationale, l'apparente dislocation, signifient donc, au premier chef, une réorganisation majeure du pouvoir économique au sein de l'union économique canadienne. Il faut examiner ceci de plus près.

Le Québec se trouve actuellement dans une union économique de type fédéral. Le degré d'intégration économique est donc, malgré les imperfections notées plus haut, beaucoup plus important que dans l'union économique et monétaire vers laquelle semble se porter le choix du Parti québécois «puisqu'à l'Union douanière, économique et monétaire, s'ajoute la dimension d'une association politique où un gouvernement central commun en relation directe avec la population est chargé des questions qui intéressent l'ensemble des unités composantes, États ou Provinces. Un partage des compétences économiques entre un gouvernement central et des gouvernement régionaux ou provinciaux s'établit sous l'autorité d'une Constitution ${ }^{25}$.»

Sur un plan global, les avantages économiques de l'intégration ne sont pas négligeables à cause notamment des économies d'échelle réalisées à tous les niveaux d'un vaste ensemble. Mais il y a aussi des coûts ${ }^{26}$. Si l'on peut supposer, selon une recette connue, un équilibre entre les avantages et les coûts qui rend acceptable l'intégration économique, on peut supposer aussi que ces coûts puissent être beaucoup trop considérables pour le développement si se superposent «dépendance économique, subordination politique et oppression nationale ${ }^{27} »$.

En fait, le contentieux des effets positifs et négatifs de l'Union fédérale sur le développement du Québec figure en bonne place parmi les cartouches

25. Commission de l'unité canadienne, Se retrouver: observations et recommandations, p. 72.

26. «Le coût de l'intégration peut être décrit comme étant essentiellement social et politique. Lorsque l'association n'a pas dépassé le palier de l'Union douanière, il y a déjà des coûts tels qu'une aptitude moindre de la part des unités constituantes, à influencer les décisions des entreprises privées, un accès plus restreint à des importations à bon marché qui n'entrent pas en concurrence directe avec la production régionale et une possibilité réduite de promouvoir l'emploi local au moyen de barrières tarifaires. En outre, tout degré supérieur d'intégration économique impose des contraintes additionnelles à l'autonomie de l'unité régionale. Celle-ci perd de sa capacité de gérer son économie puisqu'elle n'est plus autorisée à restreindre le mouvement de ses citoyens, de ses capitaux et de ses biens. Elle doit assumer le coût social de cette mobilité accrue de main-d'œuvre. De plus, les priorités de l'entité régionale peuvent être infléchies par l'existence de politiques communes qui ne tiennent pas suffisamment compte des réalités régionales $\%$. Commission de l'unité canadienne, p. 74.

27. Pour reprendre le titre de l'article de Paul R. Bélanger et Céline Saint-Pierre. 
du gouvernement provincial ${ }^{28}$. Nous avons parlé plus haut du «style unitaire de gestion" de la politique monétaire. Il faudrait discuter ici des effets régionaux des politiques de stabilisation budgétaire, de l'orientation des dépenses du gouvernement fédéral au Québec plus tournées vers le soutien des revenus (mesures sociales) que vers l'investissement productif générateur d'emplois, sans parler des politiques de la main d'œuvre ou des retombées au Québec du Pacte de l'auto. À l'inverse on pourrait évidemment citer les avantages d'un vaste marché où encore les subventions fédérales à l'importation de pétrole, mais il reste que l'écart se creuse considérablement entre le Québec et l'Ontario et que ces inégalités régionales entre les deux provinces les plus industrialisées du Canada, s'accentueront de façon dramatique si les industries de main-d'œuvre continuent à s'effondrer. En effet, nous l'avons déjà mentionné, les secteurs à faible productivité, du meuble, du textile traditionnel, de la bonneterie, du vêtement et du cuir, menacés par la nouvelle concurrence internationale, emploie le quart de la main-d'œuvre québécoise.

Depuis 1970 , toutes les études indiquent l'urgence d'une transformation radicale de l'armature industrielle du Québec et de nouvelles filières de production ont été identifiées ${ }^{29}$.

Pourtant, malgré les avantages qu'une Union fédérale peut offrir «lorsqu'une région a besoin d'un effort considérable d'adaptation économique par les transferts de ressources entre les régions ${ }^{30}$, l'État central et la grande bourgeoisie canadienne apparaissent incapables de résoudre à la fois la question du redéploiement industriel et celui des inégalités régionales. Nous en avons déjà énumiéré les raisons: bourgeoisie industrielle canadienne très affaiblie par le capital étranger, capacité très réduite en matière de recherche-développement, concentration élevée du capital, internationalisation du capital canadien et donc délocalisation de certaines activités, État libéral qui répugne malgré la situation de dépendance, à assurer un rôle de leadership en matière de développement industriel... ${ }^{31}$

De son côté, le capital régional privé québécois, dont une partie déclinera, s'il ne réussit pas sa reconversion, ne peut espérer prendre le relais et tenter de s'affirmer comme agent central de développement pour plusieurs raisons. La première tient à sa faiblesse comparativement au capital canadien et arnéricain. La seconde tient à sa différenciation nationale et ethnique qui ne permet une cohésion que sur des points limités et généralement sur une base défensive (par exemple, contre l'intervention directe de l'État dans l'écono-

28. On peut, entre autres, se reporter au requisitoire prononcé par Yves Bérubé, ministre des Terres et Forêts et Richesses naturelles du Québec, où il indique, par exemple, que «la politique monétaire canadienne a joué 6 fois contre le Québec dans les six plus importants cycles économiques connus au Canada depuis une vingtaine d'année. Ce second outil est plutôt utilisé par Ottawa pour protéger l'industrie ontarienne...» reportage de Marie-Agnès Thellier, “Ottawa utilise mal ses outils \%, le Devoir, 10 juin 1978, p. 11.

29. Sur les filières de production reposant sur les richesses naturelles, on peut se reporter à Yvon Jauron et al., Filières de production et développement régional.

30. Commission de l'unité canadienne, Se retrouver, p. 81 .

31. Voir, par exemple, la volonté du nouveau gouvernement conservateur fédéral de démanteler Petro-Canada, société d'État, au second rang des compagnies pétrolières implantées au Canada avec 3,35 milliards d'actifs. 
mie). La troisième raison concerne la dépendance à la fois économique et politique du capital régional privé québécois par rapport aux fractions hégémoniques de la bourgeoisie canadienne. En dehors du mouvement coopératif, dont le mode de contrôle est, par définition, très spécifique, ce capital ne peut globalement se définir comme externe à la bourgeoisie canadienne et ne peut donc se donner une unité et un projet spécifique. Si l'on se restreint au capital francophone, on peut évidemment identifier une série de centres de pouvoir relativement importants, qui peut s'allonger si l'on rajoute les sociétés d'État (parfois puissantes comme l'Hydro-Québec, mais en définitive assez peu nombreuses). Cependant, il ne faut pas oublier comme le font trop d'auteurs désireux de voir absolument «la main» d'une grande bourgeoisie francophone (ne s'agit-il pas plutôt d'une haute bourgeoisie limitée?) dans l'affirmation nationale, que seulement $5 \%$ des entreprises industrielles ayant plus de 50 millions de dollars d'actifs implantées au Québec appartenaient en 1974 à des Canadiens français tandis que les banques sous contrôle francophone représentent moins de $10 \%$ des actifs bancaires canadiens. En réalité, la faiblesse du capital canadienfrançais est partie intégrante de la question nationale car l'enjeu du contrôle de l'accumulation lui est sous-jacent. Il ne suffit pas, en effet, de dire, comme Gilles Bourque, que le projet péquiste vise à «créer le maximum de conditions favorables au développement du capitalisme régional québécois... à réserver l'espace économico-social le plus large possible au capitalisme régional québécois et secondairement à la nouvelle petite bourgeoisie ${ }^{32} »$. En effet, on ne peut faire abstraction du fait que l'orientation étatiste du projet implique une lutte contre la dépendance économique, une intervention directe sur le développement qui laisse un certain espace aux débats et aux choix politiques, même si ce n'est pas l'instauration d'une société socialiste dont les multiples visages possibles sont loin d'avoir été discutés pour l'instant au Québec, faute d'un mouvement de masse encore à construire.

En fait, ce sont des catégories sociales liées aux appareils de l'État québécois, qui sur la base d'un renforcement décisif de son pouvoir économique, espèrent venir à bout des problèmes que nous venons d'évoquer. Aussi la lutte pour la réorganisation du pouvoir se déroule-t-elle d'abord et avant tout, entre, d'une part, les couches dirigeantes des appareils de l'État québécois, qui, nous l'avons vu plus haut, assument par l'intermédiaire de brillants représentants, un rôle de leadership au sein du Parti québécois, et d'autre part, la grande bourgeoisie canadienne associée aux «sommets» de l'État fédéral. Ceci ne signifie évidemment pas, comme le montre Louis Maheu dans son article, que seules ces couches sociales sont concernées. Mais du point de vue de la structure du pouvoir, ce sont ces groupes sociaux qui seront les plus affectés par l'issue des affrontements en cours, avec comme enjeu, le contrôle et l'orientation de l'accumulation au Québec.

La souveraineté-association, sur le plan institutionnel, remet d'abord en cause la domination politique, et donc l'intervention directe au Québec d'un gouvernement central en relation, elle aussi directe, avec la population par le

32. Gilles Bourque, «La nouvelle trahison des clercs», dans le Devoir, $2^{\mathrm{e}}$ partie, 9 janvier 1979, p. 5. 
biais d'élections fédérales qui légitiment ses politiques sans égard à leur signification régionale ou nationale. Les problèmes québécois devenant compétence première, sinon exclusive du gouvernement du Québec, ses champs d'intervention s'élargiraient de façon considérable en même temps que s'accroîtrait son pouvoir. Par ailleurs, et l'on touche ici un point très important, la souveraineté-association imposerait une représentation directe de la haute fonction publique québécoise dans les institutions qui prendraient forme avec l'Union économique et monétaire, notamment au sein de l'éventuelle Banque centrale de l'Union Canada-Québec dont parle Vély Leroy.

Ce n'est évidemment un secret pour personne de rappeler que les ministères fédéraux, à vocation économique sont, de façon traditionnelle monopolisés par les Anglo-canadiens ${ }^{33}$. Ceci ne va pas, bien sûr, sans susciter des frustrations importantes. Mais, il y a plus, l'État québécois se trouve, aujourd'hui, être le centre de pouvoir principal de la société québécoise francophone. Son développement, ses programmes jouent depuis 20 ans, un rôle fondamental dans le dyramisme et l'affirmation de cette société. Or, malgré un engagement de plus en plus puissant dans l'économie du Québec au niveau des revenus, des dépenses, et des politiques (énergie, richesses naturelles, par exemple), ses champs de responsabilité et de pouvoir économiques restent résiduels. Sur le plan du pouvoir, l'enjeu d'une représentation directe est donc considérable pour la haute fonction publique. En lui-même, le refus de la Commission de l'unité canadienne d'envisager une participation des gouvernements provinciaux au sein des principales institutions économiques fédérales indique combien cet enjeu est crucial. Parue dans le journal le Devoir, la libre-opinion d'un fonctionnaire québécois, sur les lacunes économiques du rapport de la commission Pépin-Robarts est sans équivoque à ce sujet :

Alinsi, on ne peut laisser passer l'absence de représentation des gouvernements provinciaux à l'intérieur des principales institutions économiques. C'est pourtant de là que part le pouvoir économique. C'est dans ces lieux que se décident les politiques. Et lorsqu'on y est absent, c'est automatique, la relève vient des mêmes milieux, milieux qui ne sont pas les nôtres. Bref, on est coupé du principal pouvoir, le pouvoir de l'information. Alors que le gouvernement du Québec demande une participation, une représentation d'égal à égal dans ces institutions, la commission Pépin-Robarts ne suggère même pas un embryon de représentation ${ }^{34}$.

Sii une véritable réorganisation du pouvoir économique d'État émerge des luttes en cours, on ne doit pas supposer que l'État québécois se contentera du mode d'intervention dans l'économie de l'État fédéral actuel, surtout si les forces nationalistes se maintiennent au pouvoir. La bureaucratie, en effet, cherchera à affirmer son leadership en matière de développement par une intervention de plus en plus directe, «entrepreneuriale» pourrait-on dire, notamment dans les cas où le capital privé ou coopératif se révèle défaillant, tout en incor-

33. Le rapport du Commissaire aux langues officielles (fédéral) indique, qu'en 1978, on trouvait à la haute direction du ministère des Finances, 3 francophones sur 33 personnes, la proportion s'élève à $3,6 \%$ au ministère de la Consommation et des Corporations, $11,5 \%$ à l'Industrie et au Commerce, etc. Dans le Devoir, 21 février 1979, p. 2.

34. Jacques Boucher, «Les lacunes économiques du rapport Pepin-Robarts », dans le Devoir, 9 mars 1979 , p. 5 . 
porant la bourgeoisie régionale à son projet. En régime capitaliste et dans une société dépendante, l'État national ne semble pas disposer de nombreuses possibilités. La différence avec une situation de domination totale, c'est qu'il peut différencier ses appuis au capital en jouant sur plusieurs formes et stratégies économiques: affermissement de la bourgeoisie régionale classique, développement du secteur coopératif dans les branches où c'est possible, création et élargissement de sociétés d'État, «joint-ventures» avec le capital étranger, grignotage des maillons les plus faibles du capital étranger, renégociation de la dépendance dans l'exploitation des ressources naturelles.

L'exemple de la stratégie actuelle du gouvernement péquiste dans le secteur des richesses naturelles illustre assez bien ce mode d'intervention qui joue notamment sur le degré d'intégration des entreprises et des branches industrielles à l'ensemble québécois.

L'industrie des pâtes et papiers est bien intégrée à l'économie québécoise, achète ses équipements chez nous, alors que l'industrie de l'amiante est cataloguée comme «une industrie qui refuse de s'intégrer» et qui maintient traditionnellement une attitude d'occupant... la stratégie du gouvernement est donc opposée dans ces deux secteurs: dans les pâtes et papiers, le gouvernement a déblayé le chemin devant les entreprises, s'attaque aux problèmes de coûts, réduit les risques et propose un plan quinquennal misant sur les entreprises rentables et sur un accroissement de la productivité. Au contraire, dans le secteur de l'amiante, le gouvernement n'hésitera pas à s'immiscer directement, à prendre le leadership par l'acquisition d'Asbestos Corporation, sans désespérer d'arriver à des «ententes de développement " avec le reste de l'industrie pour transformer au Québec $12 \%$ de l'amiante québécois d'ici 10 ans $^{35}$.

Par ailleurs, dans le cadre d'un débat à l'Assemblée nationale sur la place occupée par les sociétés d'État à l'intérieur de la stratégie de développement du Québec, le ministre d'État au développement économique Bernard Landry après avoir déclaré que «la domination étrangère constitue un grand déséquilibre de l'économie», affirmait : "Si un grand projet minier doit voir le jour au Québec, il n'est pas question que les québécois ne soient pas impliqués de façon significative dans le capital-actions et la gestion [...] les sociétés d'État pourront se mobiliser à la demande du gouvernement et devenir un élément stratégique majeur ${ }^{36}$.»

\section{LA SOUVERAINETÉ-ASSOCIATION ET L'INTÉGRATION AU CENTRE}

Si l'on ne sait pas jusqu'où ira la réorganisation du pouvoir économique d'État, il n'est pas possible par contre d'imaginer le maintien du statu quo constitutionnel. Le mouvement national devrait donc obtenir sous une forme ou une autre, à court ou à moyen terme, un renforcement des pouvoirs économiques de l'État québécois.

35. Interview d'Yves Bérubé, ministre des Terres et Forêts et des Richesses naturelles, par Marie-Agnès Thellier, dans le Devoir, 21 juin 1979, p. 28.

36. Reportage de Gilles Gauthier, dans la Presse, 19 mai 1979, p. E 10. 
Cependant, le processus de différenciation nationale, dont on vient de décrire les principales dimensions, se poursuit en même temps que s'accomplit l'intégration continentale dépendante du Canada.

Aussi doit-on s'interroger, malgré le peu d'espace qui nous reste, sur l'interaction entre la réorganisation des rapports Canada-Québec et cette intégration, même s'il est encore trop tôt pour répondre réellement à ces questions étant donné les multiples scénarios imaginables et les changements qui interviendront dans les rapports de force sociaux. Il convient, en particulier, d'examiner l'hypothèse de Pierre Fournier qui voit la souverainetéassociation comme

Une stratégie de transition à moyen terme, visant à faciliter le passage vers l'option nord-américaine, et non pas comme une simple tentative de réaménager le pouvoir économique canadien ${ }^{37} \ldots \mathrm{L}^{\prime}$ ' option américaine» doit être considérée comme l'objectif ultime du Parti québécois. Il ne s'agit pas de renégocier le pacte fédéral, mais bien de négocier directement avec les USA les termes de la dépendance. Cette stratégie implique entre autres, le renforcement du contrôle sur le marché interne, la pénétration cles branches «intermédiaires» et une tentative d'accentuer les retombées locales du capital américain ${ }^{38}$.

Ainsi «l'autonomisation et le renforcement du pouvoir économique québécois aboutiraient à faire un pas de plus dans le sens de l'intégration directe à l'ensemble nord-américain ${ }^{39} »$. La double dépendance du Québec céderait alors la place à une dépendance simple, directe par rapport aux États-Unis. Pierre Fournier se plaçant dans le très long terme, considère l'association avec le reste du Canada comme une étape de transition «à cause de la très forte intégration et complémentarité des économies ontariennes et québécoises. L'intégration a beau fonctionner à l'avantage de l'Ontario, le capital québécois est trop faible et trop peu structuré pour passer outre au modus vivendi avec le capital canadien et à la période de transition qu'implique la souveraineté-association ${ }^{40}$ ».

Comme nous l'avons montré plus haut, l'intégration continentale dépendante constitue une tendance extrêmement lourde de l'évolution économique canadienne, et il est douteux que le Québec puisse renverser ce courant dans lequel il est très engagé. On peut facilement imaginer aussi qu'un Québec isolé ne résisterait probablement pas très longtemps à l'absorption par les ÉtatsUnis, comme d'ailleurs le Canada anglais qui ne dispose pas d'une identité culturelle aussi prononcée. Ainsi, le refus du Canada anglais de s'associer à un Québec souverain (peu probable cependant, compte tenu des interrelations Québec-Ontario) conduirait à la véritable dissolution de l'ensemble canadien.

Si cette éventualité doit être envisagée de façon sérieuse à cause de ses conséquences, on voit mal, par contre, pourquoi le Québec sous l'égide d'un

37. Pierre Fournier, «La Souveraineté-association, une stratégie de transition? », $1^{\text {re }}$ partie, dans le Devoir, 29 mars 1979, p. 5.

38. Ibid., $2^{\mathrm{e}}$ partie, dans le Devoir, 31 mars 1979, p. 5.

39. Ibid

40. Ibid. 
parti nationaliste irait se donner pieds et poings liés aux États-Unis. De ce point de vue, la recherche de «l'autonomisation et du renforcement du pouvoir économique québécois », c'est à dire la recherche d'une maitrise plus grande du processus et du produit de l'accumulation, notamment par une intervention accrue de l'État, contredit l'hypothèse du renforcement de l'intégration dépendante aux États Unis même s'il ne signifie pas la fin de la dépendance. Il est utile de savoir qu'en 1974, 63,1 \% des exportations internationales du Québec allaient aux États-Unis ( $80,1 \%$ pour l'Ontario). Par ailleurs, à la même date, $68,9 \%$ de nos matières non travaillées, non comestibles et près de $44 \%$ de nos matières brutes non comestibles ${ }^{41}$ étaient expédiées aux États-Unis. Alors est-il donc si contraire aux intérêts du Québec de chercher à renégocier, et directement s'il le faut, les termes de cette dépendance, tout en freinant son accentuation puisqu'elle n'a cessé d'augmenter depuis 1965 ?

Pour Pierre Fournier, l'option péquiste est clairement nord-américaine, sinon «étatsunienne»: «le rapport de forces imposera une solution plus canadienne au Parti québécois, mais ce n'est pas inévitable, et ce n'est pas non plus l'objectif du gouvernement actuel». L'option fédérale serait-elle donc strictement «Canadian» et pas nord-américaine? Les faits notés plus haut démontrent largement le contraire. Pierre Fournier prête des objectifs machiavéliques au P.Q. sans donner une démonstration suffisamment convaincante ${ }^{42}$, d'une part qu'il s'agit des objectifs du Parti québécois et d'autre part que d'autres liens avec les économies du Centre ne seront pas établis. Il est beaucoup plus probable, en fait, qu'un nouvel ensemble Canada-Québec s'il parvenait à se réaliser s'orienterait vers une diversification de ses liens économiques pour faire contrepoids à l'influence américaine. Les études commandées par le gouvernement du Québec à Luc-Normand Tellier sur «les possibilités d'un rapprochement entre le Québec, le Canada et les pays scandinaves» et à Kimon Valaskakis sur «la plausibilité d'une association Québec/Canada/Europe» indiquent plutôt que les scénarios en présence sont multiples et que les choix sont encore loin d'être faits puisque pour l'instant aucune table de négociation n'est encore en place. Tôt ou tard, il faudra pourtant en dresser une et l'histoire contemporaine du Québec par le mouvement de dislocation-intégration qui s'y produit pour contrer la marginalisation donnera des indications profondes sur l'issue possible des mouvements nationaux dans les pays industrialisés à la fin du $\mathrm{xx}^{\mathrm{e}}$ siècle.

La logique du nouveau système capitaliste mondial conduit, certes, à l'abandon d'une politique autonome de développement, mais la montée des mouvements nationaux indique l'existence de limites sérieuses à l'intégration dépendante au Canada comme en Europe.

41. GAMMA, sous la direction de Kimon Valaskakis, Prospective socio-économique du Québec, $1^{\text {re }}$ étape, Sous-Système extérieur (3), Rapport Synthèse, Québec, Office de planification et de développement du Québec, 1977, p. 56.

42. On peut se reporter aussi à la critique de Luc-Normand Tellier, "Le Parti québécois ne cherche pas l'intégration au capital américain», dans le Devoir, 5 avril 1979, p. 5. 


\section{BIBLIOGRAPHIE}

AMIN, S., l'Accumulation à l'échelle mondiale, Paris, Anthropos, 1971.

AMIN, S., le Développement inégal, Paris, Minuit, 1973.

BARNET, R. et R. MÜLLER, Global Reach: The Power of the Multinational Corporations, New Y.ork, Simon and Schuster, 1974.

BÉLANGER, P. R. et C. SAINT-PIERRE, «Dépendance économique, subordination politique et oppression nationale : le Québec, 1960-1977», dans Sociologie et sociétés, vol. 10, $\mathrm{n}^{\circ} 2$, cctobre 1978.

BOUCHER, J., "Les lacunes économiques du rapport Pepin-Robarts», dans le Devoir, 9 mars 1979, p. 5.

BOURQUE, G., «Le Parti québécois dans les rapports de classe», dans Politique aujourd'hui, $\mathrm{r} !^{\circ} 7-8,1978$.

BOURQUE, G., «La nouvelle trahison des clercs», dans le Devoir, 8 et 9 janvier 1979, p. 5 .

BRAUDEL, F., la Méditerranée et le monde méditerranéen à l'époque de Philippe II, 2 vol., Faris, Armand Colin, 1966.

BRAUDEL, F., Capitalism and Material Life, 1400-1800, London, Fontana-Collins, 1974.

DE BRUNHOFF, S., État et capital: recherches sur la politique économique, Grenoble, Presses Universitaires de Grenoble et Maspero, 1976.

CHORINEY, H., "Regional Underdevelopment and Cultural Decay", dans Saul, J. et Heron, C., Imperialism, Nationalism and Canada, Toronto, New Hogton Press, 1977.

CLEMJENT, W., The Canadian Corporate Elite, Toronto, McClelland and Stewart, 1975.

CLEMIENT, W., Continental Corporate Power: Economic Linkages between Canada and the United States, Toronto, McClelland and Stewart, 1977.

CLEMIENT, W., "Uneven Development: Canada and the World System", Communication au gie congrès mondial de Sociologie, groupe Economie et société, Uppsala, Suède, 1978. Ce texte a paru dans The New Zealand and Australia Journal of Sociology.

Commission royale d'enquête sur les groupements de sociétés (rapport de la) édité par Approvisionnements et Services Canada, 1978.

Commission de l'Unité canadienne, Se retrouver: observations et recommandations, Ottawa, Approvisionnements et Services Canada, 1979.

DUBET, F., «Sur l'analyse sociologique du mouvement occitan», dans Sociologie du travail, 3/76.

FOURINIER, P., «Le Parti québécois et la conjoncture économique au Québec», dans Politique aujourd'hui, nos 7-8, 1978.

FOURINIER, P., «La Souveraineté-association: une stratégie de transition?, dans le Devoir, 29, 30 et 31 mars 1979 , p. 5.

FURTADO, C. «Le Capitalisme post-national», dans Esprit, avril 1975 et mai 1975.

GAGNON, G. et L. MAHEU (éditeurs), «Changement social et rapports de classes», Numéro thématique de la revue Sociologie et sociétés, vol. 10, $\mathbf{n}^{\circ} 2$, octobre 1978.

HALARY, Ch., M. LAGANA, C. SAINT-PIERRE (éditeurs), "Québec, de l'indépendance au socialisme », numéro spécial de Politique aujourd'hui, nos 7-8, 1978.

HOLOHAN, W., «Le conflit du Larzac: chronique et essai d'analyse», dans Sociologie du travail, $3 / 76$.

JAURON, Y., N. ALEXANDRE, J.-Y. LAVOIE, L., NADEAU, R. CORBEIL, Filières de production et développement régional, Office de planification et de développement du Québec, «Études et recherches», 1977.

LAURIN-FRÉNETTE, N., Production de l'État et formes de la nation, Montréal, Nouvelle Optique, 1978.

LEROY, V., la Question monétaire en rapport avec le Québec: l'actualité générale du problème sous le régime politique fédéral et les perspectives issues du projet de Souveraineté-associaiion. Rapport pour le ministère des Affaires intergouvernementales du Québec, Québec, 任diteur officiel du Québec, 1978.

MASSIEY, D., «Regionalism : Some Current Issues», dans Capital and Class, $\mathrm{n}^{\circ}$ 6, automne 1978.

MICHALET, Ch. A., le Capitalisme mondial, Paris, P.U.F., 1976.

MURRAY, V., le Parti québécois : de la fondation à la prise du pouvoir, Montréal Hurtubise, H.M.H., 1976.

MURRAY, V., «Le Parti québécois: les tensions au sein de l'alliance indépendantiste», dans Politique aujourd'hui, nos 7-8, 1978.

NAYLOR, T., "The Rise and Fall of the Third Commercial Empire of the Saint Lawrence », dans 1G. Teeple (édit.), Capitalism and the National Question in Canada, Toronto, University of Toronto Press, 1972.

NAYLOR, T., The History of Canadian Business, 1867-1914, Toronto, James Lorimer and Co., 1975.

PALLOIX, Ch., l'Économie mondiale capitaliste, Paris, Maspero, 1971. 
PALLOIX, Ch., les Firmes multinationales et le procès d'internationalisation, Paris, Maspero, 1973.

QUÉRE, L., Jeux interdits à la frontière, Paris, Anthropos, 1978.

RAY, D. M., "The Location of American Subsidiaries in Canada», Economic Geography, vol. XLVII, ${ }^{\circ} 3$, juillet 1971, pp. 389-400.

RAY, D. M., "Regional Economic Development and the location of U.S. Subsidiaries», Communication à l'Incentives Location and Regional Development Conference organisée par le Gouvernement du Manitoba, janvier 1975.

SALES, A., la Bourgeoisie industrielle au Québec, Montréal, Presses de l'Université de Montréal, 1979.

STOPFORD, J. M., et L. T. WELLS, Managing the Multinational Entreprise, London, Longman, 1972.

SUNKEL, O. et E. FUENZALIDA, «Transnational Capitalism and National Development», dans J. J. Villamil, Transnational Capitalism and National Development, London, Harvester Press, 1979.

TELLIER, L. N., «Le Parti québécois ne cherche pas l'intégration au capital américain», dans le Devoir, 5 avril 1979, p. 5.

VALASKAKIS, K., l' "Option Europe», analyse de la plausibilité d'une association Québec/ Canada/Europe, Québec, ministère des Affaires intergouvernementales, 1978.

VERNON, R., Sovereignty at Bay, New York, Basic Books, 1971.

VERNON, R., les Conséquences économiques et politiques des entreprises multinationales, Paris, Robert Laffont, 1974.

WALLERSTEIN, I., The Modern World System, New York, Academic Press, 1974.

WILKINS, M., The Emergence of Multinational Entreprise: American Business Abroad from the Colonial Era to 1914, Cambridge, Harvard University Press, 1970.

WILKINS, M. The Maturing of Multinational Enterprise: American Business Abroad from 1914 to 1970, Cambridge, Harvard University Press, 1974. 


\section{RESUMÉ}

Cet article met en rapport deux mouvements apparemment contradictoires qui contribuent à donner sa configuration au monde contemporain : d'une part un mouvement de globalisation, qui conduit à parler de système capitaliste mondial, d'autre part un mouvement de différenciation nationale qui, sortant du Tiers-monde, touche maintenant les grands pays industrialisés et les menace de morcellement. S'appuyant sur l'exemple Québec/Canada, l'auteur montre d'abord comment l'intégration continentale dépendante, l'internationalisation et la concentration qui constituent les dimensions principales du processus de globalisation, empêchent le Canada de se maintenir comme champ économique autonome et surtout comme unité pertinente pour résoudre la question du redéploiement industriel et du développement régional inégal. C'est dans ce contexte que le mouvement national québécois, fondé d'abord sur la lutte contre des structures de domination/subordination entre deux nations, pose à travers la revendication d'une «souveraineté-association", la doutle question de la différenciation nationale et de l'intégration au système global pour contrer la marginalisation, l'affrontement pour la réorganisation du pouvoir social ayant surtout lieu entre les couches dirigeantes de l'État québécois et la bourgeoisie canadienne associée aux sommets de l'État fédéral.

\section{SUMM.ARY}

This article defines a relationship between two apparently contradictory movements which contribute to the configuration of the contemporary world : on one hand a movement toward globalization, which brings us to the world capitalist system, and on the other hand a movement of national differentiation which, having originated in the Third World, is now affecting the great industrialized countries and is threatening to bring about their break-up. Using the example of Quebec/Canada, the author shows first how dependent continental integration, internationalization and concentration, that make up the main dimensions of the process of globalization, prevent Canada from holding, its own as an autonomous economic unit, and especially as a meaningful unit for solving the problem of stimulating renewed industrial growth and of uneven regional development. It is in this context that the Quebec national movement, founded in the first place on the struggle against domination/subordination structures between two nations, poses, through the demand for "sovereignty-association", the double question of national differentiation and integration into the global system as a means of fighting against marginalization, the clash for the reorganization of social power taking place mainly between the ruling classes of the Quebec State and the Canadian bourgeoisie associated with the higher levels of the Federal State.

\section{RESUMEN}

Este artículo pone en relación movimientos aparentementes contradictorios que contribuyen a dar su configuración al mundo contemporáneo : de una parte un movimiento de globalisación, que conduce a hablar de sistema capitalista mundial, de otra parte un movimiento de diferenciación nacional que emergiendo del tercer mundo, toca ahora los grandes países industrialisados y los amenaza de división. Apoyandose sobre el ejemplo Québec/Canada, el autor mostrara, en primer lugar como la integración continental dependiente, la internacionalisación y la concentración, que constituyen las dimensiones principales del proceso de globalisación, impiden al Canada de mantenerse como campo económico autónomo y sobre todo como unidad pertinente para resolver la cuestión del redespliegue industrial y del desarrollo regional desigual. Es en este contexto que el movimiento nacional québécois fundado en primer lugar en la lucha contre las estructuras de dominación/subordinación entre dos naciones plantea a través la revindicación de una "soberaniaasociación» la doble cuestión de la diferenciación nacionale y de la integración al sistema global para oponerse a la marginalisación, al afrontamiento en la reorganisación del poder social, que se da sobre todo entre las capas dirigentes del Estado québécois, y la burguesia canadiense asociada a las cumbres del Estado federal. 